\section{Perinatal psychiatry: East is East and West is West}

SIR: I have a particular empathy with Dr Aderibigbe and colleagues (BJP, November 1993, 163, 645-650) because of their successful research task of assessing 277 Nigerian women attending a booking-in antenatal clinic in Ibadan and then reassessing 162 women at eight weeks post-partum. Their sample size and follow-up rate were very close indeed to the Ugandan study carried out almost 20 years ago (Cox, 1979, 1983); in this study, 263 women were interviewed at the booking-in clinic and 183 reinterviewed at three months post-partum. A control group of non-pregnant, non-puerperal women was also personally interviewed.

The Nigerian researchers found that anxiety and fear were the predominant moods during pregnancy, but such moods did not accurately predict those who would develop a postnatal psychiatric disorder. In the Ugandan study, the inter-rater reliability using the Luganda translation of the Goldberg SemiStructured Interview was reported. The Nigerians were similarly careful to validate the General Health Questionnaire (GHQ) for use during pregnancy on a sample of West African women.

The differences between those two studies are of interest. The East African sample included a sizeable proportion of single or separated women $(14.8 \%$, $9.5 \%$ ), whereas only two Nigerians were in these categories. The separated Ugandans were more at risk of antenatal psychiatric disorder, but no such difference was found in the puerperium. Ugandan women lost to follow-up were more likely to have experienced psychiatric symptoms during pregnancy, to be living alone, and to have attended late in pregnancy. The Nigerians who defaulted were less likely to have psychiatric morbidity than those successfully followed up. Did the researchers have a reputation for treating antenatal psychiatric morbidity? What proportion of disturbed mothers during the perinatal period was treated by the research team?

Of the Nigerian women, $30 \%$ were 'cases' at the first prenatal assessment - a high prevalence of psychiatric morbidity, yet similar to that reported for Ugandans by Assael et al (1972). The reduction in prevalence of psychiatric morbidity from before to after childbirth, with only a limited overlap between these two populations, is similar to that found in Uganda. Can the authors provide data on the proportion of women who scored above threshold on the GHQ depression subscale? It would be fascinating if they can indicate the frequency of postpartum as well as antenatal depression found in their study.
These studies from Central Africa have implications for the training of health workers on that continent, as well as for researchers and clinicians in Europe. Collaboration with social anthropologists in future prospective studies of perinatal mental illness is likely to be particularly revealing.

Assael, M. I., Namboze, J. M., German, G. A., el al (1972) Psychiatric disturbances during pregnancy in a rural group of African women. Social Science and Medicine, 6, 387-395.

Cox, J. L. (1979) Psychiatric morbidity and pregnancy: controlled study of 263 semi-rural Ugandan women. British Journal of Psychiatry, 134, 401-405.

(1983) Postnatal depression: a comparison of African and Scottish women. Social Psychiatry, 18, 25-28.

Keele University

JoHN L. Cox Staffordshire

\section{An attachment school of psychotherapy}

SIR: Dr Holmes (BJP, October 1993, 163, 430-438) notes that John Bowlby's attachment theory is a rationale and a biological basis for psychotherapy. He states that there is no attachment school of psychotherapy, although it provides an underpinning which is applicable to all psychotherapies.

I would like to inform Dr Holmes about interpersonal psychotherapy (IPT), which was developed by my late husband, Gerald Klerman, myself and several collaborators, and which rests heavily on Bowlby's attachment theory. In our book, Interpersonal Psychotherapy of Depression (New York: Basic Books, 1984), we quote heavily from Bowlby's seminal work and note that "the concept of attachment bonds provides a strong theoretical basis for understanding the interpersonal context of depression and developing psychotherapeutic strategies to correct distortions produced by faulty attachments in childhood" (pp. 52-53). Moreover, we note that attachment theory has stimulated a strong body of empirical research on the association between interpersonal relationships and depression, and we review some of this work.

Interpersonal psychotherapy is relatively well known. The manual has been translated into Italian and Japanese, and was used in a clinical trial of bulimia in which staff at Oxford University participated. There have been two completed controlled clinical trials of IPT for acute major depression, two for maintenance treatment, and one in geriatric depression. Many modifications of IPT have been developed and are being tested, including those for depressed adolescents, depressed pregnant women, depressed patients who are HIV positive, for 